\title{
ESTIMACIÓN DE LA PÉRDIDA DE SUELOS POR EROSIÓN HÍDRICA EN LA CUENCA DEL RÍO SIGUAS UTILIZANDO GEOINFORMATICA
}

\author{
ESTIMATED LOSS OF SOIL BY EROSION WATER IN THE SIGUAS RIVER BASIN USING \\ GEOINFORMATIC
}

${ }^{1}$ Domingo M. Portuguez M.

\begin{abstract}
Resumen
Los modelos de erosión conjuntamente con las aplicaciones de Sistemas de Información Geográfica (SIG) constituyen herramientas eficaces para los estudios de la pérdida de suelo por erosión, el mismo que constituye un problema medioambiental grave. En el presente trabajo se pretende mostrar el gran potencial de los SIG, a través de la implementación de una aplicación práctica, para el estudio de la erosión en la parte alta de la cuenca del río Siguas en Arequipa. Se utilizó el modelo USLE (Universal Soil Loss Equation) como método para el cálculo de las tasas de pérdida de suelo, posibilitando la elaboración de una cartografía de estados erosivos, que permite la identificación de las áreas de especial sensibilidad a la erosión.
\end{abstract}

Palabras Clave: erosión en cuencas, USLE, sistemas de información geográfica, modelo digital de elevación.

\begin{abstract}
Erosion models together with applications of Geographic Information Systems (GIS) are effective tools for the study of soil loss by erosion that it is a serious environmental problem. This research assesses to the potential of GIS for erosion evaluation, applied to study trhe erosion in the upper reaches of the river in Arequipa Siguas. Erosion is evaluated using the USLE model (Universal Soil Loss Equation) to calculate soil loss rates, this data is the base for creating erosive states cartography that allows the identification of the areas most susceptible to erosion.
\end{abstract}

Key words: soil erosion, USLE, GIS, digital elevation model.

\section{Introducción}

En nuestro país, principalmente en las partes altas que corresponden a la región de la sierra, el principal factor de degradación del suelo es la erosión hídrica. El daño generado por esta erosión en zonas montañosas, de pendientes irregulares y escarpadas se hace cada vez más evidente, debido a que los fenómenos climáticos son más intensos e irregulares. El suelo agrícola dañado va perdiendo su capacidad productiva, generándose por consiguiente problemas económicos, sociales y ecológicos.

Este trabajo tiene como principal objetivo mostrar el estudio y análisis de problemas ambientales mediante técnicas SIG. La aplicación de una metodología de trabajo basada en SIG, necesaria para el estudio de la erosión hídrica así como la estimación de la pérdida de suelo por erosión hídrica en la Cuenca del río Siguas en Arequipa, a través de la implementación práctica de un método conocido como la Ecuación Universal de Pérdida de Suelo (USLE).

\section{Materiales y métodos}

Información Cartográfica: Carta Nacional (Escala: 1/100000), mapa ubicación espacial de las estaciones. (Escala: 1/100000), mapa cobertura y uso. (Escala: 1/50000), mapa de suelo. (Escala: 1/50000).
Información de Precipitación: Precipitaciones media anual (20 años de registro)

Software: Software Arc GIS, extensiones: 3D Analyst for ArcGIS y Spatial Analyst for ArcGIS

Área de estudio: La cuenca del río Siguas forma parte hidrográfica de la cuenca Quilca, se encuentra ubicada políticamente entre las provincias de Arequipa, Caylloma y Majes en la Región Arequipa - Perú, coordenadas Este 148 000/ 215000 Norte 8157 500/ 8258650 , entre 4670 y 150 m.s.n.m. (Fig. 1). El río Siguas se forma al confluir los ríos Lluta y Lihualla, que al unirse con el río Vitor forman el río Quilca, que desemboca al Océano Pacifico. Tiene como fuentes de alimentación los deshielos de los Nevados Ampato y Sabancaya y parte del Hualca Hualca y Ananto, y las precipitaciones pluviales de las partes altas de la cuenca. Hasta su confluencia con el río Vítor, tiene un área de $1774 \mathrm{~km}^{2}$; su cuenca húmeda, hasta la cota $3000 \mathrm{msnm}$, es de $765 \mathrm{~km}^{2}$. Entre los 3000 y 3700 msnm se presenta agricultura tradicional en los sectores de Querque, Lluta, Taya y Huanca. 


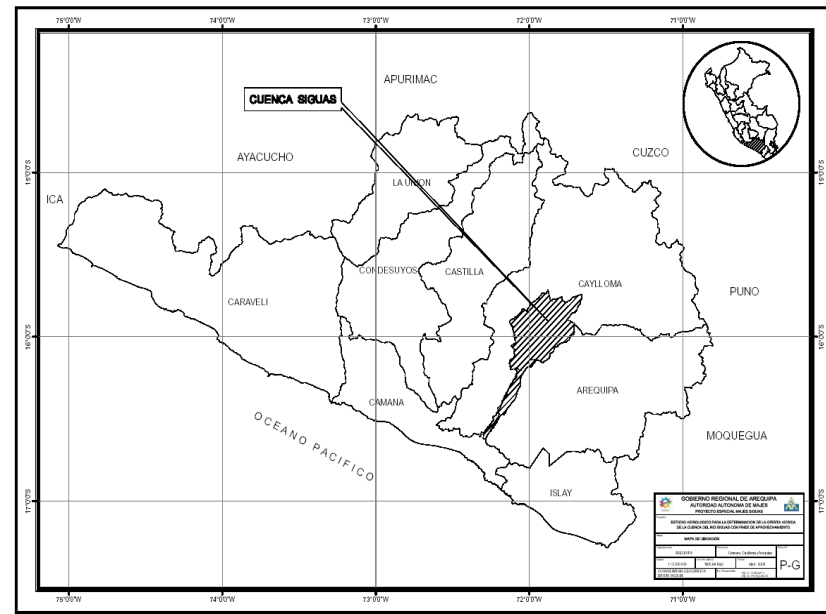

Figura 1. Mapa de ubicación de la cuenca del río Siguas.

La cuenca del río Siguas está conformado por la sub cuenca de alto Siguas donde los suelos presentan relieves topográficos ligeramente inclinados (2-10\%), mayoritariamente superficiales, poco profundos que han ido perdiendo su capa arable debido a su sistema de riego, la erosión ocasionada por las lluvias y topografía. Presentan ligera pedregosidad y buen contenido de materia orgánica. La sub cuenca bajo Siguas presenta suelos cuyas pendientes fluctúan de 0 a $5 \%$, de textura predominantemente franco-arenosos, con alto contenido de materia orgánica. Los suelos ubicados en las partes bajas y colindantes con el río presentan problemas de drenaje y salinidad debido a las filtraciones provenientes de la Irrigación Majes, por lo que en estas áreas sólo crecen pastos naturales (ANA, 2008).

Determinación de la pérdida de suelo por erosión hídrica: Para la aplicación del método denominado Ecuación Universal de Pérdida de Suelos de Wischmeier y Smith (1978) se procedió de acuerdo al esquema de la Fig. 2. La expresión más conocida de esta ecuación, es:

$$
\mathrm{A}=\text { R. K. S. L. C. P }
$$

Donde A es la pérdida de suelo por unidad de superficie (tn/ha.año), R es el factor lluvia o índice de erosión pluvial (MJ. mm /ha h), K es el factor erodabilidad del suelo (tn. ha.h/ha.MJ.mm), L es el factor longitud de pendiente (adimensional), $\mathrm{S}$ es la pendiente (adimensional), $\mathrm{C}$ es el factor cultivo y/o ordenación y $\mathrm{P}$ es el factor prácticas de cultivo.

Yataco (2007) refiere que el factor $\mathrm{R}$ no puede ser modificado, $\mathrm{K}$ depende fuertemente de la textura de los suelos, lo que no puede ser fácilmente mejorada. Sólo C, P y LS pueden ser cambiados, las prácticas agronómicas comunes, pueden utilizarse para modificar la cobertura vegetal, y consecuentemente $\mathrm{C}$, mediante manejo de desechos y mejoramiento de los sistemas de siembra, el factor P puede ser cambiado por prácticas agronómicas, como las barreras vegetativas o con obras de conservación de suelos como terrazas, zanjas o acequias de ladera, la modificación del factor $\mathrm{P}$ está íntimamente ligado a la reducción de la longitud de pendiente (L) y la pendiente (S) sólo puede ser modificada mediante obras de conformación del terreno, tales como terrazas.

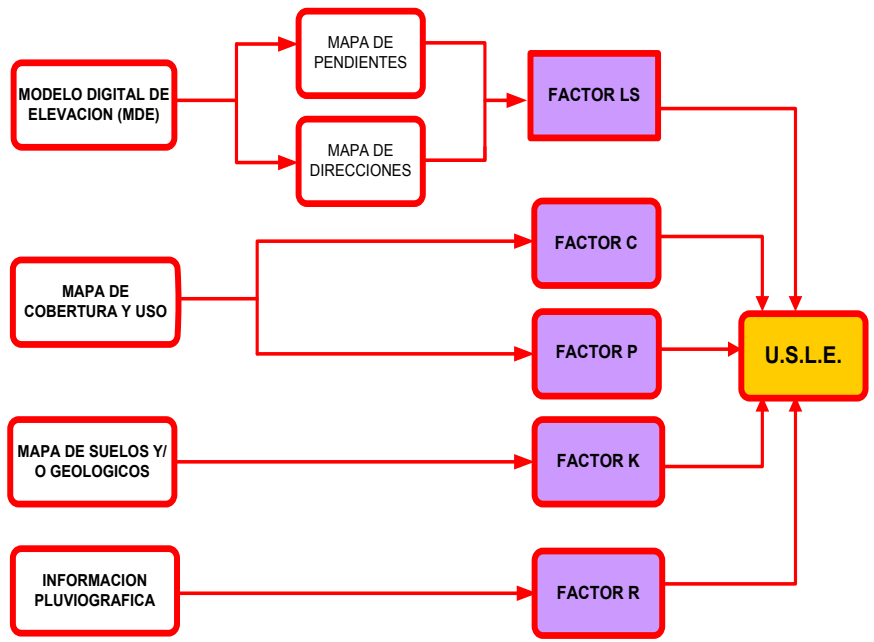

Figura 2. Modelo conceptual para la estimación de los niveles de pérdida de suelo según el método USLE.

\section{Determinación de los factores de la Ecuación Universal de Pérdida del Suelo}

a) Factor R: Roose en 1995, citado por Ramos (2001) calculó el factor $\mathrm{R}$, mediante la siguiente ecuación:

$R=1.73 * 0.5 * P$

Donde $\mathrm{P}=$ Precipitación media anual, generándose el mapa de isoerodientes, del cual se obtiene la superficie que representa el factor $\mathrm{R}$.

b) Factor LS: USLE tiene en cuenta la topografía del terreno mediante la introducción del factor L de longitud de la pendiente del terreno y del factor $\mathrm{S}$ de grado de la pendiente. Para la elaboración de un mapa de pendientes, en el marco de un Sistema de Información Geográfica, se requiere un modelo digital del terreno MDT o DEM (Digital Elevation Model). Ramos (2001) cita en la Tabla 1 , los valores que asigna directamente a una ladera el valor del factor L.S, elaborado por la Facultad de Hidráulica e Hidrología de la Escuela Técnica Superior de Ingenieros de Montes (Madrid).

Tabla 1. Valores del Factor L.S. para diferentes pendientes.

\begin{tabular}{cc}
\hline $\begin{array}{c}\text { Grupos de pendientes } \\
\text { en } \%\end{array}$ & Factor \\
\hline $0-3$ & L.S \\
$3-12$ & 0,3 \\
$12-18$ & 1,5 \\
$18-24$ & 3,4 \\
$24-30$ & 5,6 \\
$30-60$ & 8,7 \\
$60-70$ & 14,6 \\
$70-100$ & 20,2 \\
$>100$ & 25,2 \\
\hline
\end{tabular}


c) Factor K: Indica la erodabilidad del suelo o susceptibilidad a la erosión hídrica del suelo. La variable $\mathrm{K}$ se obtiene a partir del mapa geológico.

d) Factor C: referido a la protección de la cobertura vegetal al suelo cuando intercepta las gotas de lluvia amortiguando su energía de impacto disminuyendo el efecto erosivo, se determina a partir del mapa temático de cobertura, determinándose los valores de $\mathrm{C}$ en el mapa. En el presente trabajo se han usado los valores (Tabla 2) publicados por Wischmeier y Smith (1978) que definen los valores de $\mathrm{C}$ para los tipos más comunes de cultivos, así como para las diferentes coberturas.

e) Factor P: Se consideró el valor de este factor igual a 1, como lo refiere Rodríguez (2007) cuando no es posible el conocimiento de estas prácticas de control de la erosión ni de manejo de las actividades productivas.

Tabla 2. Valores de cobertura vegetal (C).

\begin{tabular}{lc}
\hline \multicolumn{1}{c}{ Cultivo y Práctica } & $\begin{array}{c}\text { Media anual } \\
\text { del factor C }\end{array}$ \\
\hline Suelo desnudo & 1.0 \\
Bosque o matorral denso (alto mulch) & 0.001 \\
Pradera herbácea en buenas condiciones & 0.01 \\
Pradera sobrepastoreada & 0.1 \\
Maíz, sorgo, alto rendto., laboreo & $0.20-0.55$ \\
convencional & \\
Maíz, sorgo, alto rendto.,sin laboreo & $0.50-0.90$ \\
convencional & $0.02-0.10$ \\
Maíz, sorgo, bajo rendto.,laboreo mínimo & \\
Maíz, sorgo, alto rendto., laboreo con & $0.12-0.20$ \\
chisel & $0.30-0.45$ \\
Maíz, sorgo, bajo rendto., laboreo con & $0.40-0.70$ \\
chisel & $0.01-0.025$ \\
Algodón & $0.20-0.50$ \\
Pradera herbácea & $0.10-0.40$ \\
Soya & $0.10-0.20$ \\
Trigo & $0.30-0.80$ \\
Arroz & $0.01-0.8$ \\
Cacahuate & 0.56 \\
Frutales con cobertura vegetal & $0.001-0.003$ \\
Caña de azúcar & $0.003-0.009$ \\
Arbolado denso & 0.041 \\
Arbolado forestal clareado & $0.003-0,013$ \\
Arbolado muy clareado (25-60\%) & $0.20-0.013$ \\
Matorral con buena cobertura & 0.25 \\
Matorral ralo y eriales & 0.15 \\
Cultivos anuales y herbáceos & 0.003 \\
Pasturas & 0.035 \\
Plantas herbáceas y matojos (100\%) & $0.15-0.09$ \\
Plantas herbáceas y matojos (60\%) & 0.45 \\
Cubierta escasa (60\%) & \\
Cubierta inapreciable & \\
\hline & \\
&
\end{tabular}

Estados erosivos: Mármol (2006) cita la Tabla 3 para clasificar los mapas de erosión por clases o grados de erosión que tiene como referencia la clasificación de la FAO (1980).
Aplicación de la Ecuación Universal de Pérdida de Suelos (USLE): Para la elaboración del mapa de estados erosivos según el modelo USLE para la cuenca del rio Siguas (Arequipa) se ha procedido a la superposición de mapas (análisis espacial) de los mapas elaborados con los factores reseñados anteriormente (K, L.S, C, R).

Tabla 3. Tolerancias establecidas por FAO-PNUMAUNESCO.

\begin{tabular}{lc}
\hline Clases de erosión & $\begin{array}{c}\text { Pérdida de suelos (tn/ha/ } \\
\text { año) }\end{array}$ \\
\hline Nula a ligera & menor a 10 \\
moderada & $10-50$ \\
Alta & $50-200$ \\
Muy alta & mayor a 200 \\
\hline
\end{tabular}

\section{Resultados y discusiones}

La aplicación de los SIG permiten la estimación cuantitativa de pérdida de suelos (Congreso Internacional de Ingeniería Gráfica, 2002), esta información, permite orientar la toma de decisiones políticas a gran escala (ordenamiento territorial para la conservación y el desarrollo) ya que los resultados obtenidos indican donde y en qué cantidad se produce el fenómeno erosivo (Orue et al, 2007), posibilitando conocer la localización espacial del fenómeno y su intensidad. Rodríguez (2007). Mati et al (2000), emplearon la metodología (SIG) para calcular la pérdida de suelo y mapear el riesgo de erosión en forma interactiva comparándolos con los valores de pérdida de suelo medidos en parcelas de erosión de la cuenca del Alto Ewaso Ng'iro North de Kenya, obteniendo resultados similares.

La erosividad de la lluvia, en la cuenca del rio Siguas (R) oscila entre 8.65 a $337.35 \mathrm{MJ} \mathrm{mm} / \mathrm{ha} \mathrm{h}$. El régimen pluviométrico que registran las lluvias se caracteriza por su irregularidad temporal de mayor frecuencia e intensidad entre los meses de diciembre a abril mientras que el resto del año es baja considerándose casi nula en los meses de junio a agosto. Los valores de precipitaciones promedio anuales dentro de la cuenca están principalmente entre $1.8 \mathrm{~mm}$ y $710 \mathrm{~mm}$, valores que se consideran clasificados como de baja erosividad (Almoza et al, 2007).

El factor de erodabilidad del suelo $(\mathrm{K})$ se obtuvo a partir del mapa geológico, Rodríguez (2007) refiere que las unidades litológicas obtenidas a partir de la digitalización de los mapas geológicos, permiten una reclasificación para identificar las mismas con su valor del factor $\mathrm{K}$ y obtener así la distribución espacial de la erodabilidad de los suelos. En la cuenca Siguas, los valores del factor $\mathrm{K}$ están comprendidos entre 0.28 y 0.48 t.ha.h/ha.MJ. $\mathrm{mm}$, siendo el valor predominante en el ámbito de la cuenca $\mathrm{K}=0.48$ t.ha.h/ha.MJ.mm, que corresponde a conglomerados y arenas aluviales, que corresponde a suelos moderadamente susceptibles al desprendimiento.

Para la determinación del factor LS, se inicia interpolando las isolineas del mapa topográfico por el método de 
triangulación, con el software Arc GIS para obtener Modelo Digital de Elevación (MDE) del que se obtuvo un mapa de pendientes (utilizando la herramienta Slope de Arc GIS) que refleja los distintos valores del factor S. Por otra parte, el factor L se promedió para asignarlo a un gradiente de pendientes (tabla 1), obteniendo finalmente los valores del factor LS para la zona de estudio (Fig. 7). El $48.6 \%$ de la extensión de la cuenca presenta valores de factor topográfico (LS) de 8.7 cuyas pendiente corresponden a suelos entre 24 a $30 \%$, y $1.58 \%$ de la extensión de la cuenca presenta valores de este factor de 25.2, que corresponden a suelos con pendientes más pronunciadas entre 70 a $100 \%$ (Fig. 4).

$\mathrm{El}$ factor $\mathrm{C}$ se refiere a la protección que la cobertura vegetal proporciona al suelo al interceptar las gotas de lluvia y amortiguar su energía de impacto y de escorrentía disminuyendo el efecto erosivo. Los valores del factor C se obtuvieron de los valores de la Tabla 2, obteniendo el mapa con la distribución espacial del factor C (Fig. 6) a partir del mapa de cobertura y uso.

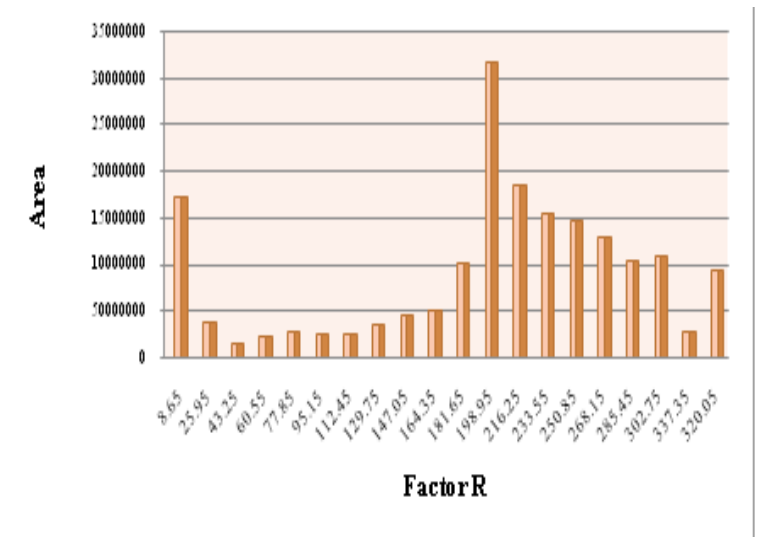

Figura 3. Valores del Factor R en la Cuenca Siguas.

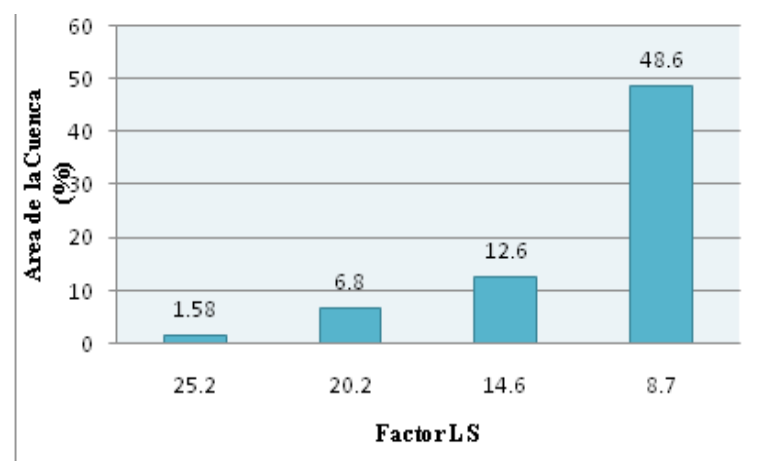

Figura 4. Valores del Factor LS en la Cuenca Siguas.

Una vez obtenidas todas las capas temáticas correspondientes a todos los factores descritos en el método USLE, se calcularon los valores de las tasas de erosión multiplicando los mapas correspondientes, obteniéndose valores de pérdida de suelo (A) que oscilan entre 0 y 1400 t/ha/año. En la Fig. 10, se muestra el mapa de estados erosivos para la zona de estudio de donde se identifica que las zonas que presentan las tasas más altas de pérdida de suelo corresponde a la zona que presenta suelos susceptibles al desprendimiento y con pendientes entre $70-100 \%$, representando a una menor extensión de la cuenca $(1.58 \%)$.

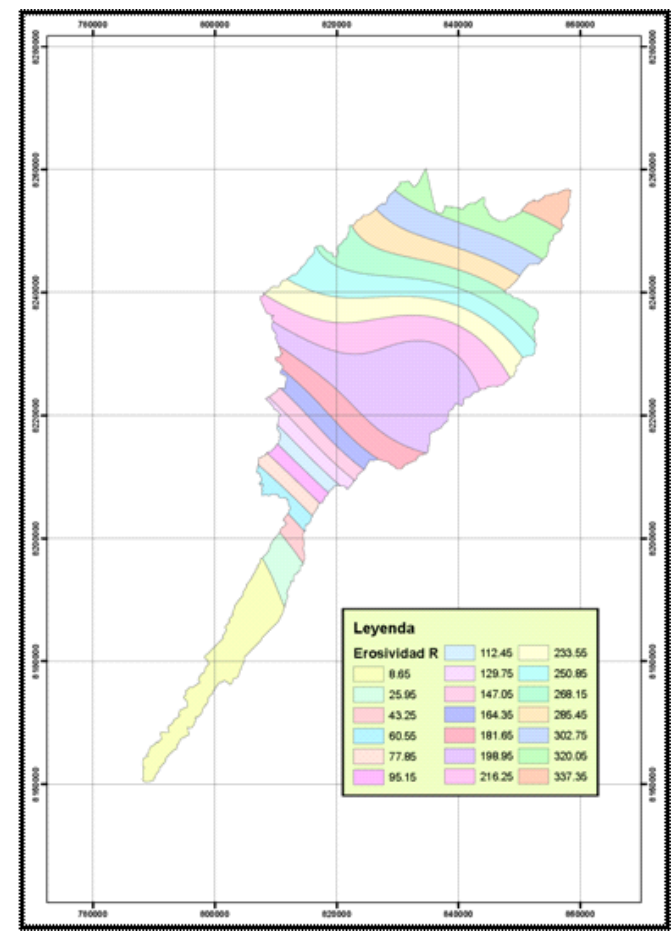

Figura 5. Mapa de Isoerodientes. Factor R en la Cuenca Siguas.

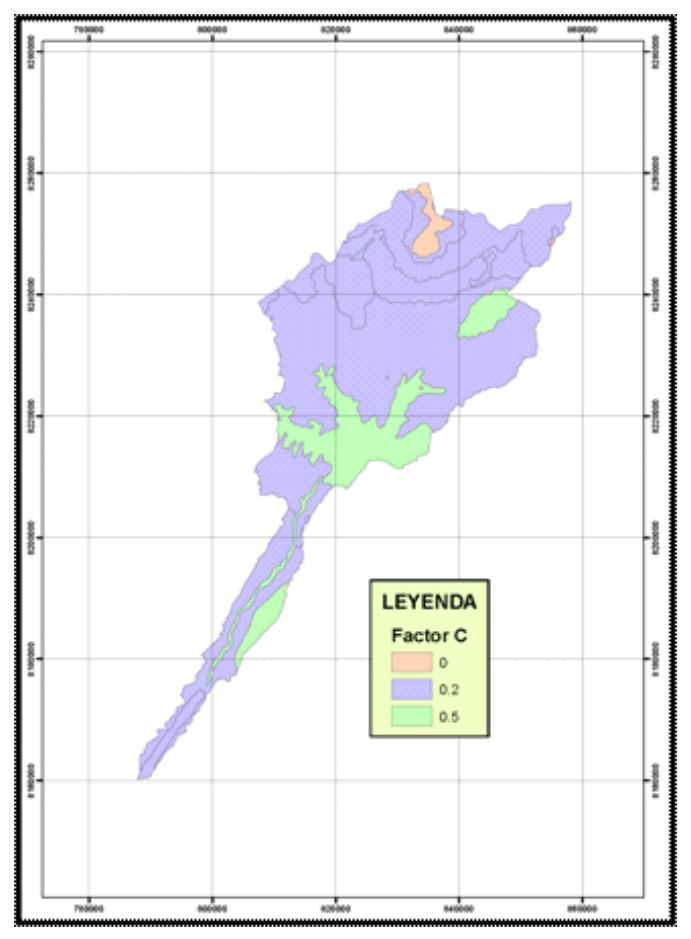

Figura 6. Mapa de Cobertura Vegetal del Suelo. Factor C en la Cuenca Siguas. 


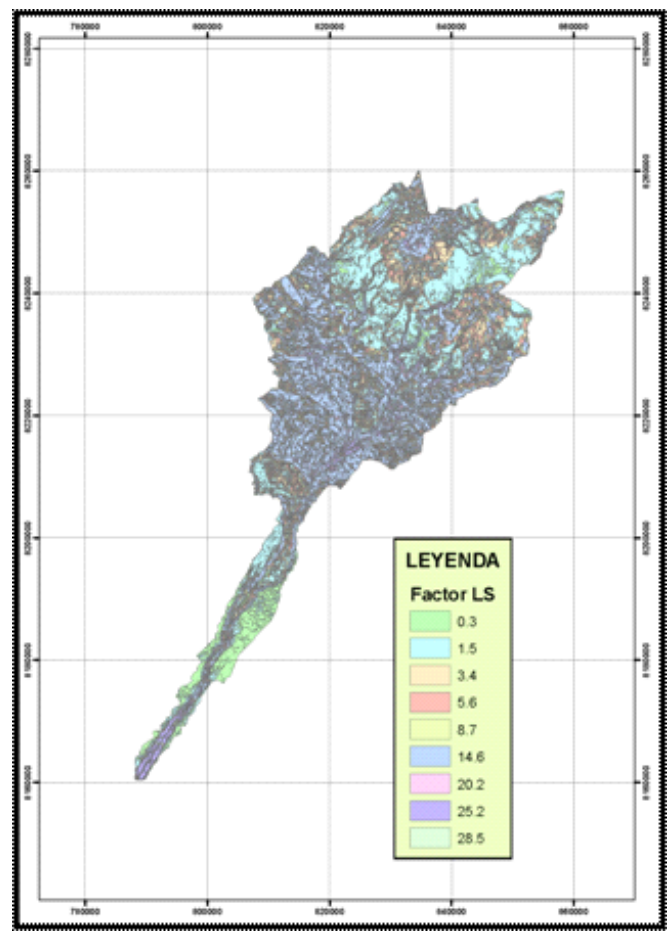

Figura 7. Mapa del Factor topográfico LS en la Cuenca Siguas.

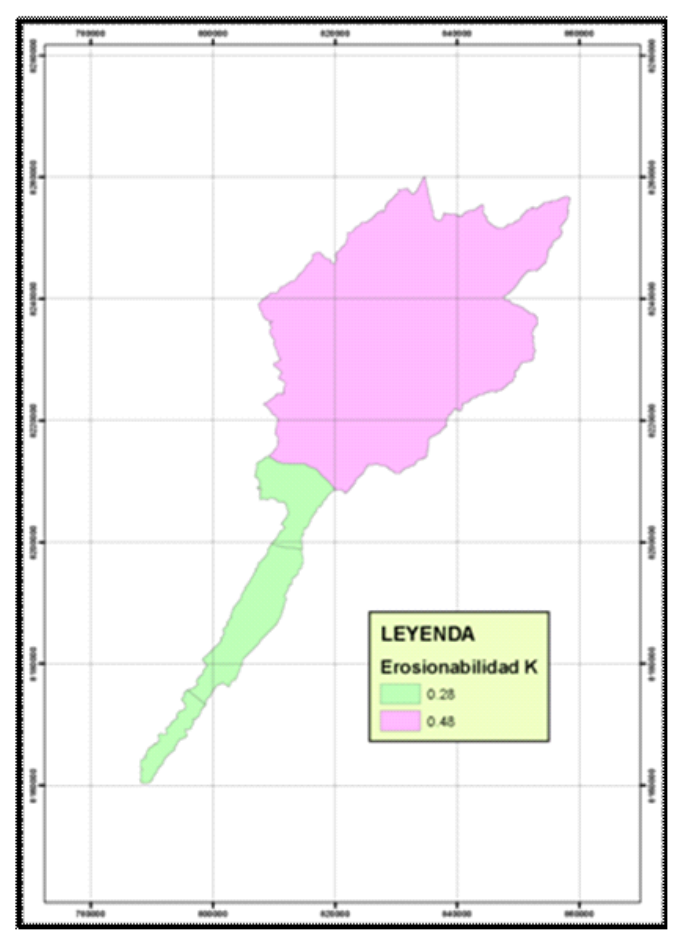

Figura 8. Mapa de erodabilidad. Factor K en la Cuenca Siguas.

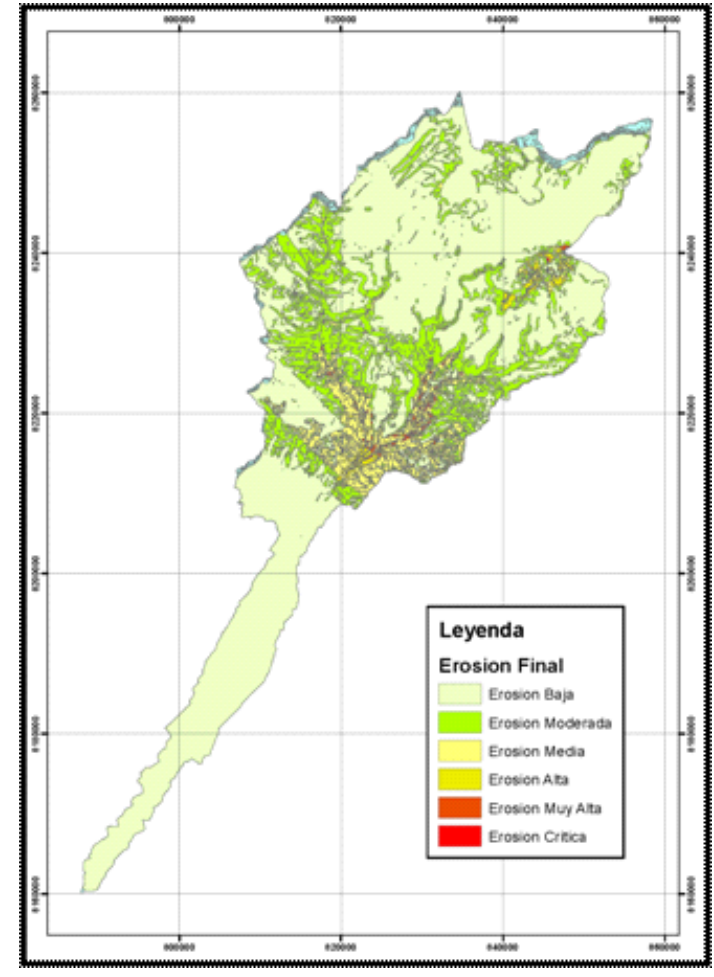

Figura 9. Mapa de estados erosivos en la Cuenca Siguas.

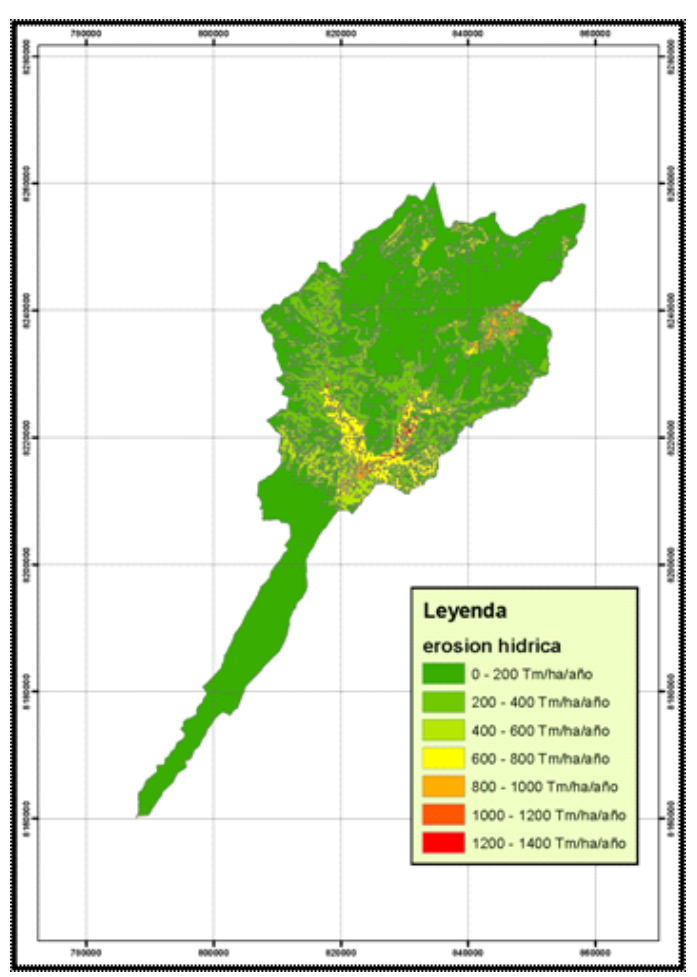

Figura 10. Mapa de Pérdida de Suelos por Erosión Hídrica. 


\section{Conclusiones}

Los valores del factor $\mathrm{R}$ obtenidos para la Cuenca del rio Siguas oscilan aproximadamente entre 8.65 a $337.35 \mathrm{MJ}$ $\mathrm{mm} / \mathrm{ha} \mathrm{h}$.

En los meses de diciembre a abril las precipitaciones pueden llegar a valores e $710 \mathrm{~mm}$, clasificándose como de baja erosividad.

Los valores del factor $\mathrm{K}$ en la cuenca Siguas, están comprendidos entre 0.28 y 0.48 t.ha.h/ha.MJ.mm. El valor predominante $\mathrm{K}=0.48$ t.ha.h/ha.MJ.mm, corresponde a suelos moderadamente susceptibles al desprendimiento, por lo tanto es un factor importante para la erosión de estos suelos.

El $48.6 \%$ de la extensión de la cuenca presenta valores de factor topográfico (LS) de 8.7 cuyas pendiente corresponden a suelos entre 24 a $30 \%$ (pendientes moderadas), mientras que el $1.58 \%$ de la extensión de la cuenca presenta suelos con pendientes más pronunciadas entre 70 a $100 \%$, que favorecen a la erosión hídrica de la zona de estudio.

Las tasas de pérdida de suelo llegan hasta 1400 t/ha/año, correspondientes a zonas de mayor pendiente y a suelos correspondientes a conglomerados y arenas aluviales, que son moderadamente susceptibles al desprendimiento.

\section{Literatura citada}

Almoza, Y.; Ruiz, M.; Medina, H. y Alonso, G. 2007. Determinación de la Erosividad de las precipitaciones en el oeste de Cuba. Zonas Áridas 11(1): 139-149.

Autoridad Nacional del Agua (ANA). 2008. Diagnostico de los problemas y conflictos en la gestión del agua en la Cuenca Chili-Quilca. Ministerio de Agricultura y Riego. República del Perú (en línea). Consultado el 27 de Setiembre del 2014. Disponible en http://www.ana.gob. pe/media/406338/caratula $\% 20 \% 20$ diagnostico $\% 20$ chili. pdf

Congreso Internacional de Ingeniería Gráfica INGEGRAF (XIV/2002, Santander - España). 2002. Determinación de riesgos de erosión en la comarca olivarera de "Sierra Mágina" (Jaén) mediante técnicas SIG y Teledetección. Actas del Congreso. Gallego F.J., Cobo M., Navarrete L., Valderrama J., Jiménez R. ISBN 84-699-8559-0 2002.

Mármol, L. 2006. Introducción al Manejo de Cuencas Hidrográficas y Corrección de Torrentes. Manejo de Cuencas Hidrográficas. Universidad Nacional de Salta. Salta. Argentina. 287 p.

Mati, B.; Morgan, R.; Gichuki F.; Quintonb J.; Brewerb, T. and Linigerd, H. 2000. Assessment of erosion hazard with the USLE and GIS: A case study of the Upper Ewaso Ng'iro North basin of Kenya. International Journal of Applied Earth Observation and Geoinformation. 2(2):78-86

Orúe, E.; Laterra, P. y Cabria, F. 2007. Expansión de la frontera agrícola en Argentina y erosión hídrica: mapas de riesgo utilizando el Modelo USLE con apoyo de SIG. Revista Teledetección-Hacia un mejor entendimiento de la dinámica global y regional (en línea). Consultado el 29 de setiembre del 2014. disponible en http://www.aet.org. es/congresos/xii/arg24.pdf.

Ramos, C. 2001. Modelamiento Ambiental para análisis de susceptibilidad erosiva en la cuenca media y alta del rio Cañete y determinación del mapa de erosión (Sistema de Información Geográfica). UNALM (en línea). Consultado el 1 de Setiembre del 2014. Disponible en http://tarwi.lamolina.edu.pe/ cramost/MODELO $\% 20$ DE\%20EROSION\%20HIDRICA\%20-\%20SIG.pdf

Rodríguez, M. Potencialidad de las técnicas SIG para la Gestión Medioambiental: Aplicación al estudio de la erosión. M+A. Revista electrónica Medio Ambiente. UCM. No 3 Mayo 2007: 76 - 89 (en línea). Consultado el 3 de diciembre de 2010. Disponible en http://www. ucm.es/info/iuca/2007\%201\%20RODRIGUEZ.pdf

Wischmeier, W. H. and Smith, D. 1978. Predicting rainfall erosion losses. A guide to conservation planning. U.S.D.A. Agriculture Handbook 537: 58 p.

Yataco, A. 2007. Evaluación cuantitativa de la erosión hídrica utilizando varillas de erosión en suelos con diferentes grados de cobertura vegetal de la comunidad campesina de Yuracmayo, Lima. Tesis Mag. Sc. Lima Perú. Escuela de Post Grado de la Universidad Nacional Agraria La Molina. 76 p. 